\title{
Discovering User Interface Requirements of Search Results for Mobile Clients by Contextual Inquiry
}

\author{
David L. Chan, Robert W.P. Luk, Hong Va Leong, and Edward K.S. Ho \\ Department of Computing, The Hong Kong Polytechnic University, Hung Hom, \\ Kowloon, Hong Kong \\ \{csdlchan, csrluk, cshleong, csksho\}@comp.polyu.edu.hk
}

\begin{abstract}
This paper reports our in situ study using contextual inquiry (CI). It solicits user requirements of hierarchically organized search results for mobile access. In our experiment, search activities of our subjects are recorded in the video, and the interviewer solicits the interface requirements, during and after the experiment. An affinity diagram is built as a summary of our findings in the experiment, and the major issues are discussed in this paper. The search behavior of our subjects is summarized into a flow chart. In this study, we report mobile interface features that are desired by our users in addition to those found in an earlier survey.
\end{abstract}

Keywords: Hierarchical access, mobile search, interface design.

\section{Introduction}

Recently, there has been much interest [1-10] in organizing search results as hierarchies. Such works can be used for semantic-based retrieval [6,7] and for mobile search (e.g., $[8,9]$ ) in order to circumvent the limitations of mobile clients (such as limited display, input constraints, bandwidth costs, etc). They focused on the technical advancement of the access structures, for examples, by building better hierarchies ([1-6]), by determining better hierarchical structures [10] or by advanced browsing using the Accordian Summarization [11]. By contrast, there are less research works in obtaining requirements of user interfaces (e.g., [12]) that access search results hierarchically. Earlier, [13,14] provided some observations to fill this gap by surveying the user preferences of user interface design of existing mobile content providers that used list and hierarchical access structures. However, surveying existing user interfaces as a means of soliciting user requirements is too restrictive, because many desired user interface features may be needed but not implemented because of: (1) financial costs, (2) subjects' unwillingness to make suggestions, (3) the absence of practical knowledge in the field. [15-19] have advocated that user requirements should be obtained by soliciting them from end-users who are directly using the interfaces in the work environment. Such in situ studies have been carried out for mobile systems in general (e.g., [20-23]), but they have not investigated the specific problem of soliciting user interface requirements of hierarchically organized search results for mobile clients. This paper addresses this problem by using an in situ study methodology, called Contextual 
Inquiry (CI), which is a component of Contextual Design [15] in user requirement solicitation. This paper discusses and analyzes practical difficulties as well as the functional requirements of searching using mobile clients based on CI.

\section{Our Work}

According to [23], it is not easy to accomplish naturalistic study. An alternative is to make direct observation by shadowing a small number of users. As a result, our data collection and analysis are mainly qualitative, and our experiments assign realistic mobile search tasks to users. We do not limit our observations to a pre-defined issue space [23] (see Table 1 for examples), and we collect a wide range of information about mobile search systems. Our analysis is based on both our observation and the user provided information.

Table 1. Example search tasks

In Yahoo, find some item that you want to buy and tell us the result.

"Existing technology used to detect diseases could also allow parents to select their babies' most important physical traits from eye color and hair color to brain power and even the shape of the babies' nose."

Please find out the full story of the news in ABC news.

Start from $\mathrm{BBC}$, find out an Israeli delegation which is due to arrive in Washington and is trying to secure an emergency aid package worth $\$ 12 \mathrm{bn}$.

Start from Internet Wire, find out "AFM Hospitality Corporation, one of North America's leading hospitality companies, (which) announced today that it has discontinued negotiation regarding the acquisition of Marshall Management, Inc. of Salisbury, Maryland."

\subsection{Set Up}

Fifteen experienced PDA users participate in our study - ten males and five females. The background of these subjects is similar to an earlier survey $[13,14]$ because some participated in the earlier survey in order to gauge their in-depth knowledge. All users have experience in human computer interaction. They are undergraduates aged 20-23, and they have modest to expert level of experience with mobile phone and Internet. On average, they have at least four years of online search experience, and the experience to access the Internet and mobile clients. They reflect the most active population using mobile clients [25], and they are expected to use mobile clients in the future.

Figure 1 shows our set up for conducting contextual inquiry on mobile search. A PDA (Pocket PC - Toshiba e740) is connected to a laptop computer that is connected to the Internet. An audio recorder and a video capturing tool record the experiment. Data traffic and user interactions are recorded by the proxy server. The Internet Explorer and Klondike WAP browser access Web and WAP based information, 


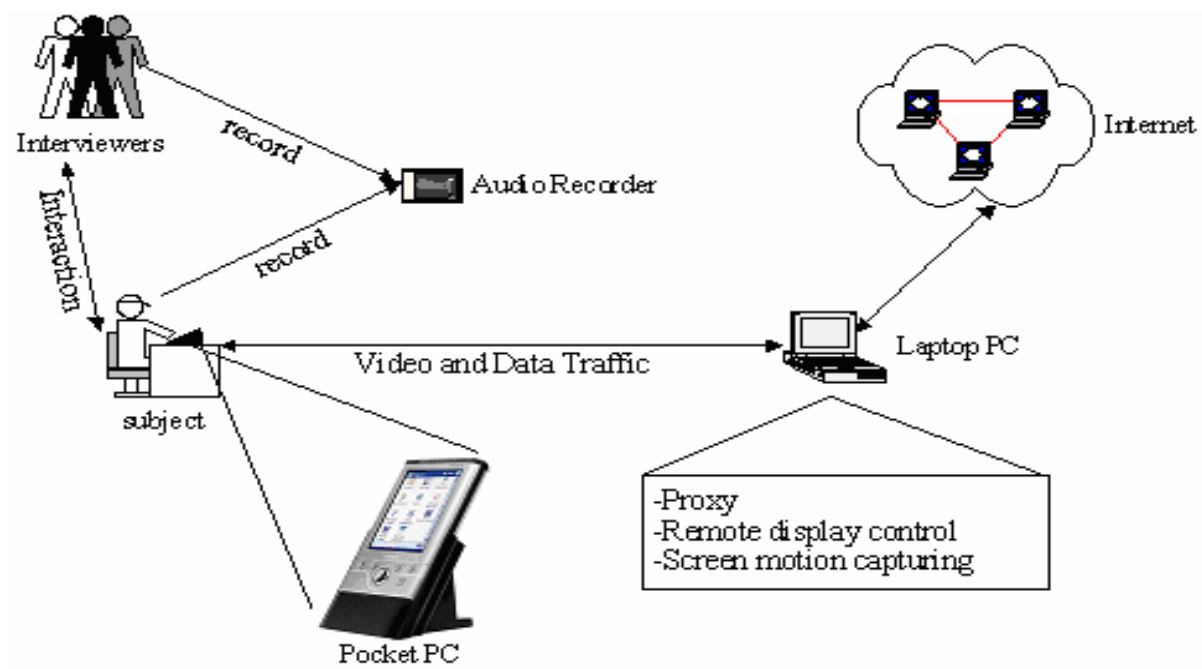

Fig. 1. Set up of Contextual Inquiry for studying mobile search

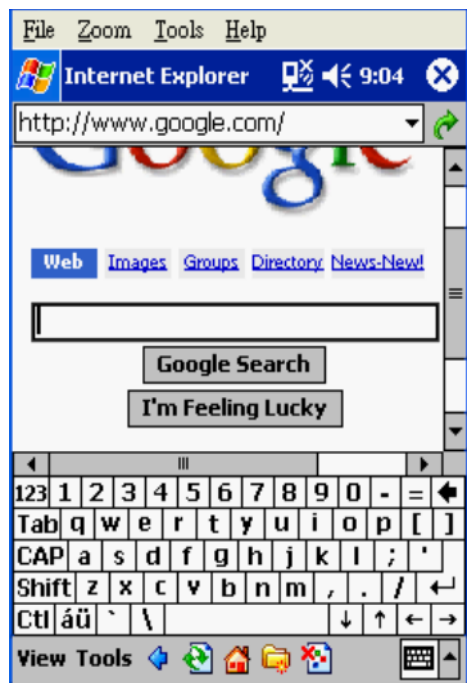

Fig. 2. Internet Explorer (Google)

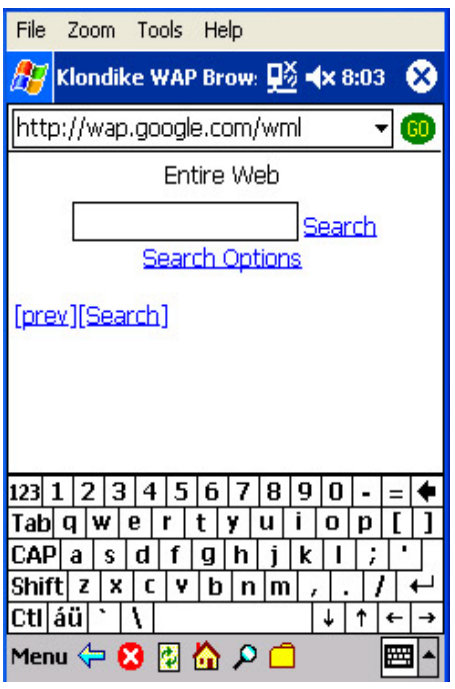

Fig. 3. Klondike WAP Browser (Google)

respectively. As the browsers' interfaces and functionalities are similar, we can reduce the influence of the interface in the experiment because of the difference in Web and WAP content.

The search tasks are designed for observing and analyzing practical user requirements, opinions, and behaviors, when the users use mobile clients to search. Users are asked to perform various Web/WAP search tasks by using keywords or hierarchy based search tools/engines. These tasks vary from general search for any 
related information items to specific search that has detailed relevance criteria (please refer to Table 1 for examples).

\subsection{Data Collection}

Each of the four interviews with users is divided into two subsections lasting for one hour each. The interviews are open ended. While the interview is in progress, an interviewer observes and discusses with users on their requirements and difficulties, as well as opinions on mobile search systems. Such information, together with users' actions and interviewer's observations, is recorded during the interview. A short discussion is conducted with the users in order to discover and wrap up problems and ambiguities after each interview which is video/audio taped as supplementary information. Additional information, such as user background, is collected with post experimental questionnaires for a better understanding of the users' behaviors.

\section{Findings}

Data are gathered from open-ended interviews, discussion and observations. As a result, users can raise their requirements, opinions and difficulties freely while they are searching via mobile clients. However, the collected data are normally unstructured and do not have a fixed focus on the users' requirements, opinions and difficulties, so it is quite difficult to draw conclusions from such data directly. Therefore, we adopt an affinity diagram to consolidate such data for analysis as in many Contextual Inquiries/Designs. An affinity diagram is a hierarchical structure that represents information from general to specific, where each of the parent nodes represents a general idea concluded from the associated child nodes. Initially, our collected data are represented by leaf nodes of the affinity diagram. Similar nodes belonging to the same layer are grouped and consolidated in order to find a general idea of each group. As a result, the affinity diagram represents grouped and concluded ideas from the collected data. Using the affinity diagram, we explore the requirements, opinions and difficulties of users by referring to those consolidated conclusions. Apart from adopting affinity diagrams, the search behavior is simplified into a flow chart.

\subsection{Affinity Diagram Summary}

The consolidated requirements are re-constructed into an affinity diagram which is itemized [24]. Due to space limit, we show an extract of the itemized affinity diagram in Figure 4. In addition, we summarize the findings from this affinity diagram and cross-reference these findings to the itemized affinity diagram labeled with $[A D$ section number] in [24].

Feasibility of hierarchical access: We assume that hierarchical access is not only a human preferred access structure, but also a suitable one for supporting mobile search in practical situations. If our assumption is valid, hierarchical access will fulfill those practical requirements mentioned in the affinity diagrams. Thus, we try to support our assumption with observations as follows. 


\subsubsection{User interface design}

1.1.2.1 Layout: Preferred layout is a sirmple, clear, tidy and sharp design with common style and static standard, in which components are clearly indicated and separated to fit on single screens by using up all the space

1.1.2.1.1 Components should be clearly indicated and separated

1.1.2.1.1.1 Catalog names/itemsititles/inks/function keys should be separated clearly

1.1.2.1.1.2 Should clearly indicate links/text

1.1.2.1.2Simple, clear, tidy and sharp design with common style and static standard

1.1.2.1.2.1 Prefer simple, clear, tidy and sharp design rather than complicated design

1.1.2.1.2.2 Prefer common style and static standard in layout and catalog design

1.1.2.1.3Better fitted on single screen and use up all the space

1.1.2.1.3.1 Use up all the space

1.1.2.1.3.1.1 Better use up all the space of the screen

1.1.2.1.3.1.2 Too much space results in give-up browsing many scrolling missing content and difficult to locate useful content

\subsection{Fitted on single screen}

\subsection{1 Information fitted on single screens supports faster searching}

1.1.2.1.3.2.2 Prefer information fitted on single screen

Fig. 4. Extracts of our affinity diagram (itemized) in [24] after minor modifications

First, owing to the limited input capacity of mobile clients, selection based input is more suitable than keyboard based input [AD 1.1.1.3.6]. This favors hierarchical access since hierarchal access input is selection based. This finding is further confirmed by [AD 1.1.1.3.7] that a local catalog (hierarchy) is preferred more than local keyword search for Web access.

Second, [AD 2.1.1.3.1] indicates that much information is not grouped or organized which results in an ambiguous layout, a slower access speed, and a waste of display area. The use of hierarchy is one way for grouping and organizing information. Users agree that hierarchy can provide a tidy layout [AD 2.1.1.6, 2.1.1.6.5.2] and prefer to use hierarchies for grouping information [AD 2.1.1.6.6.3], because hierarchies can help them to identify relevant information quickly by providing a tidy, organized layout and an effective summary [AD 2.1.2.3.1.1.5].

Third, it will be more convenient to use hierarchical access if a user knows about the search item belongs to which catalog or category in the hierarchy [AD 2.1.2.3.1.1.7.3]. In some cases, users need to use a hierarchy when they cannot specify queries or do not have a clear search objective [AD 2.1.2.3.1.1.7.1-2].

Fourth, hierarchies could make good summaries by concentrating important information which, in turn, allows users to anticipate the relevance of a group of documents when browsing only the small and organized hierarchical summary. Potentially, users access faster as mentioned in [AD 1.1.1.2.2] and can decide quickly whether to wait for loading detailed content or not as found in [AD 1.1.1.2.1.2-3].

Exploration of User Requirements: First, users need a simple, clear and tidy layout in a common or static form, where interface components are indicated clearly and fitted 
to a single screen [AD 1.1.2.1]. This is because a complicated, information-rich layout causes ambiguity because of the limited display area. Simple text-based links, wellcontrasted text (black) and clear background (white) are thought to be suitable for information display on mobile clients [AD 2.1.1.4, 1.1.2.2].

Second, users report that summarization, concentrated information, heading, indexing and local hierarchy (catalog) could be useful for them to understand, search, and identify useful content quickly and conveniently in mobile clients. This is because they experienced difficulties to browse a lot of information in a small display area using a mobile client, unlike searching using desktop computers [AD 2.1.1.7.1]. They suggested that only necessary content, such as abstract and heading, is needed in the search result, while additional useful information, such as the total number of search result and the total number of pages, may be useful [AD 2.1.1.8.2, 2.1.1.8.3].

Third, users indicate that they will be unable to search for a single purpose for more than 15 minutes if they use mobile clients to search for information in real life [AD 1.1.1.2.1.1]. Also, they could not accept slow loading and access speed, when they are searching via mobile clients [AD 1.1.1.2.2]. They suggest that pictures and information that cannot be fit onto a single page should be removed, so that the loading speed can be increased, and less scrolling is needed (because of slower accessing speed) [AD 1.1.1.2.2]. Users also find scrolling difficult to locate, capture and access information [AD -2.1.1.3.3]. They suggest that utilization of splitting pages, physical control keys and hierarchical summarization could relieve the reliance on or even replace scrolling. Thus, communication cost, access speed and loading speed as well as tidy layout are ranked at higher priorities than other functions such as pictures and scroll bars [AD 1.1.1.3.9].

Fourth, users suggest sorting search results by relevance ranking, alphabetical ordering, or user interest [AD 2.1.2.3.2]. They prefer less than 20 items in one page [AD 2.1.2.3.3.1-2].

Fifth, function keys should be unique and popular, together with descriptions clearly shown. For drawing users' attention effectively and for users' convenience, function keys should be grouped together, and put at the top and bottom of a long page (scrolling is required), or at the bottom of a single screen page [AD 2.1.1.1]. In addition, highlighting important keywords in the search results, abstracts and headings may provide useful hints to identify useful information, because users indicate that they usually pay attention to them. Also, highlighting visited links can also help users to quickly skip the visited links [AD 1.1.1.1].

Finally, users want a feasible mechanism to group information, and the use of hierarchy is one method that can help users to identify information quickly by providing a tidy display and a summary of information. Users use hierarchies, when they are unable to specify queries or do not have a clear objective for searching, or when they know exactly that the search items belong to certain parts of the hierarchy. Users accept changes to the hierarchical structure based on dynamic data (i.e. dynamic hierarchy), while (path) information provided by the hierarchy needs to make sense to users and should not be changed often. Also, they prefer detailed hierarchy and accept multiple paths that matched the same concept. This concept should make sense to users, or reflect the underlying content of the information units. For display, hierarchy and headings should not be put together. The names of nodes of catalog should be well separated in a single screen [AD 2.1.1.6]. Also, users need 
status tracing functions, like forward, backward and direct jump functions, to go to the different status or levels of the hierarchy. This is useful when users change their mind [AD 3.1.2.2].

\subsection{User Search Behavior Summary}

User behavior is recorded using video, and the behavior is summarized using a flow chart. The final analysis integrates all the users' search behavior by combining the flow charts. Because the aim of this paper is to explore issues on designing hierarchical access structure, only user behavior regarding hierarchical access is analyzed. The behavior flow chart (Figure 5) contains all the possible paths collected because it represents all the users' behavior regarding hierarchical access. Paths via pull-down menus are ignored, because pull-down menus do not belong to the hierarchical access structure. This simplifies the analysis, and it focuses on hierarchical access to search result only. Also, paths via related headings are ignored because it is not related to search results. After discarding the irrelevant user behavior, the final analysis of the user search behavior is shown in Figure 6.

The user behavior of hierarchical access on mobile client is simple and straightforward (Figure 6). There are only three major components involved, namely, catalog or hierarchy, picture/abstract/headings and content. We may further ignore the picture component because pictures are not preferred [AD 1.1.1.3.8-9]. Instead, we observe that the linkage between the above three components is important. Hence, the interface should have some device to enable users to jump to these three search behavior components in the hierarchy.

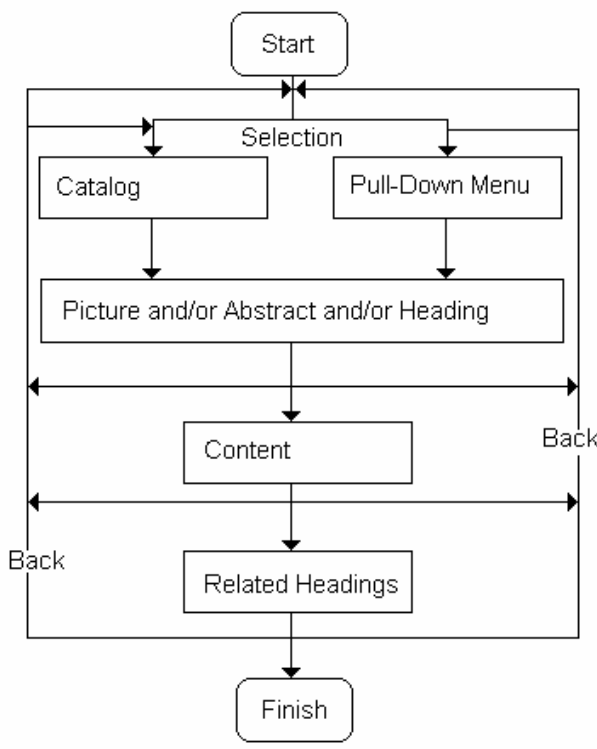

Fig. 5. Search behavior flow chart

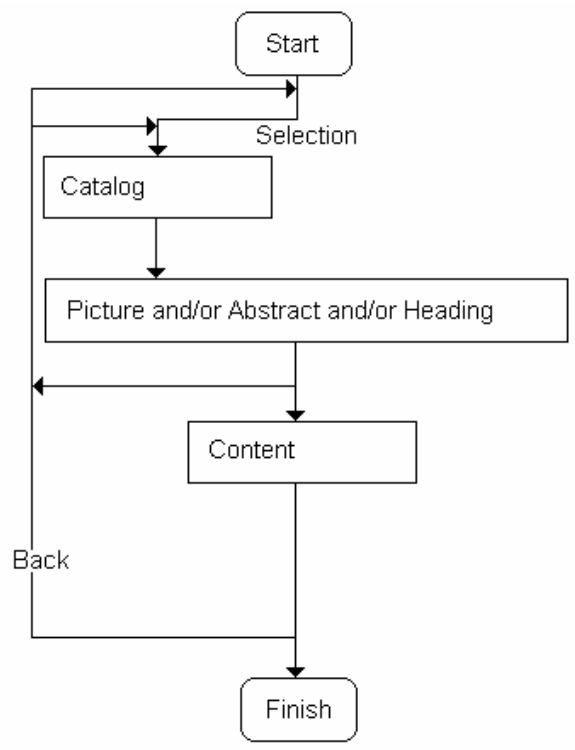

Fig. 6. Simplified search behavior flow chart 
Table 2. Features found by survey and contextual inquiry

\begin{tabular}{|c|c|}
\hline $\begin{array}{l}\text { User Preference } \\
\text { Survey }[13,14]\end{array}$ & $\begin{array}{l}\text { Contextual Inquiry } \\
\text { (In This Article) } \\
\end{array}$ \\
\hline No similar features & $\begin{array}{l}\text { Bold, underline or highlight the keywords and visited links } \\
\text { Always fit information on a single screen (i.e. avoid }\end{array}$ \\
\hline No similar features & scrolling) \\
\hline No similar features & Use selection based input \\
\hline No similar features & $\begin{array}{l}\text { Local catalog, menu and abstract to provide a summary of } \\
\text { information }\end{array}$ \\
\hline No similar features & Show description of function keys \\
\hline No similar features & Group function keys \\
\hline No similar features & Use text-based links \\
\hline No similar features & Black and white background and fonts (for good contrast) \\
\hline No similar features & Break/split long pages to avoid scrolling \\
\hline No similar features & Use physical function buttons \\
\hline No similar features & Use pull-down menu \\
\hline No similar features & $\begin{array}{l}\text { Make summary of information by heading, abstract or local } \\
\text { catalog/hierarchy }\end{array}$ \\
\hline No similar features & $\begin{array}{l}\text { Sort items by relevance ranking, alphabetical ordering or } \\
\text { user interests }\end{array}$ \\
\hline No similar features & $\begin{array}{l}\text { Show total number of search results, or available } \\
\text { documents or pages }\end{array}$ \\
\hline $\begin{array}{l}\text { Provide facility for } \\
\text { jumping back to higher } \\
\text { level }\end{array}$ & Use backward and forward functions \\
\hline $\begin{array}{l}\text { Provide facility for } \\
\text { jumping in between } \\
\text { different catalogs }\end{array}$ & $\begin{array}{l}\text { Add function for directly jumping in between different } \\
\text { status, e.g. Page number list }<1,2,3,4,5>\end{array}$ \\
\hline $\begin{array}{l}\text { Classify articles into } \\
\text { different catalogs }\end{array}$ & Categorize information \\
\hline $\begin{array}{l}\text { List titles together with } \\
\text { abstract in each of the } \\
\text { catalog }\end{array}$ & $\begin{array}{l}\text { Browsing and search result only need the abstract and } \\
\text { heading }\end{array}$ \\
\hline $\begin{array}{l}\text { Jump to the } \\
\text { next/previous document }\end{array}$ & No similar features \\
\hline $\begin{array}{l}\text { List of catalog names } \\
\text { with the related titles }\end{array}$ & No similar features \\
\hline
\end{tabular}

\section{Comparison}

Table 2 summarizes the important user interface features indicated by an earlier survey $[12,13]$ and CI. Some of the features in the survey are similar to features found by the CI. Some of the features are only indicated as important in either the user preference survey or the CI. Of particular interests are the importance of 
categorization of information (a similar important feature in the survey is "Classify articles into different catalogs"), because it supports the use of hierarchical access that is similar to catalog. To illustrate the advantage of CI, we compared the user requirements found by $\mathrm{CI}$ with those discovered by an earlier survey of mobile search interfaces. Out of a total of 24 interface features found, four similar feature pairs are discovered by both CI and the survey, 14 features by CI alone and only two features by the survey alone.

\section{Conclusion}

An affinity diagram summaries our findings in the experiment, and the major issues are discussed in this paper. The search behavior of our subjects is summarized into a flow chart. We found 14 additional mobile interface features that enrich our knowledge from an earlier survey on mobile interface design for search results.

Acknowledgements. This work is supported by the HKPU project \# G-U289.

\section{References}

1. Sanderson, M., Croft, W.B.: Deriving structure from texts. In: ACM SIGIR 1999, pp. 206213. ACM Press, New York (1999)

2. Lawrie, D., Croft, W.B.: Generating hierarchical summaries for web searches. In: ACM SIGIR 2002, pp. 457-458. ACM Press, New York (2002)

3. Lawrie, D., Croft, W.B., Rosenberg, A.: Finding topic words for hierarchical summarization. In: ACM SIGIR 2001, pp. 349-357. ACM Press, New York (2001)

4. Lawrie, D., Croft, W.B.: Discovering and comparing topic hierarchies. In: RIAO 2000, pp. 314-330 (2000)

5. Robertson, G.G., Cameron, K., Czerwinski, M., Robbins, D.C.: Polyarchy visualization: visualizing multiple intersecting hierarchies. In: CHI 2002, pp. 423-430 (2002)

6. Shadbolt, N.R., Gibbins, N., Glaser, H., Harris, S., Schraefel, M.C.: CS AKTive Space or how we stopped worrying and learned to love the Semantic Web. IEEE Intelligent Systems 19(3), 41-47 (2004)

7. Schraefel, M.C., Shadbolt, N.R., Gibbins, N., Glaser, H., Harris, S.: CS AKTive Space: Representing computer science in the semantic web. In: WWW 2004, pp. 384-392 (2004)

8. Chan, D.L., Luk, R.W.P., Mak, W.K., Leong, H.V., Ho, E.K.S., Lu, Q.: Multiple related document summary and navigation using concept hierarchies for mobile clients. In: ACM SAC, pp. 627-633 (2002)

9. Karlson, A.K., Robertson, G., Robbins, D.C., Czerwinski, M., Smith, G.: FaThumb: a facet-based interface for mobile search. In: CHI 2006, pp. 711-720 (2006)

10. Yang, C.C., Wang, F.L.: Fractal summarization for mobile devices to access large documents on the web. In: WWW 2003, pp. 215-224 (2003)

11. Buyukkokten, O., Garcia-Molina, H., Paepcke, A.: Accordion summarization for endgame browsing on PDAs and cellular phones. In: CHI 2001, pp. 213-220 (2001)

12. Capra, R., Marchionini, G., Oh, J.S., Stutzman, F., Zhang, Y.: Effects of structure and interaction style on distinct search tasks. In: JCDL 2007, pp. 442-451 (2007) 
13. Chan, D.L., Luk, R.W.P., Leong, H.V., Ho, E.K.S., Lu, Q.: A preliminary study of user interface design issues for mobile web access. In: WWW 11 Mobile Search Workshop, pp. 4-14 (2002)

14. Chan, D.L., Luk, R.W.P., Ho, E.K.S., Lu, Q.: A preliminary study on multiple documents access via mobile devices. In: Chung, C.-W., Kim, C.-k., Kim, W., Ling, T.-W., Song, K.H. (eds.) HSI 2003. LNCS, vol. 2713, pp. 116-127. Springer, Heidelberg (2003)

15. Beyer, H., Holtzblatt, K.: Contextual design: defining customer-centered systems. Morgan Kaufmann, San Francisco (1998)

16. Williams, G., McClintock, M.: Usability at Microsoft. In: CHI 1997, pp. 144-145 (1997)

17. Cleary, T.: Communicating customer information at Cabletron systems Inc. ACM Interactions 6(1), 44-49 (1999)

18. Coble, J.M., Karat, J., Kahn, M.G.: Maintaining a focus on user requirements throughout the development of clinical workstation software. In: HFCS, pp. 170-177 (1997)

19. Curtis, P., Heiserman, T., Jobusch, D., Notess, M., Webb, J.: Customer-focused design data in a large multi-site organization. In: HFCS, pp. 608-615 (1999)

20. Druin, A.: Cooperative inquiry: developing new technologies for children with children. In: HFCS, pp. 592-599 (1999)

21. Fouskas, K., Pateli, A., Spinellis, D., Virola, H.: Applying contextual inquiry for capturing end-users behavior requirements for mobile exhibition services. In: $1^{\text {st }}$ Intern. Conf. Mobile Business, CDROM (2002)

22. Nokia 9000 Communicator. PC Week, pp. 39-40 (1996)

23. Palen, L., Salzman, M.: Beyond the handset: designing for wireless communications usability. ACM Transactions on Computer-Human Interaction 9(2), 125-151 (2002)

24. Chan, L.: Supporting searching for mobile clients. M.Phil. Thesis, Department of Computing, The Hong Kong Polytechnic University (2005)

25. Video Research Ltd.: Survey on Mobile Phone Usage Situation (2000) 